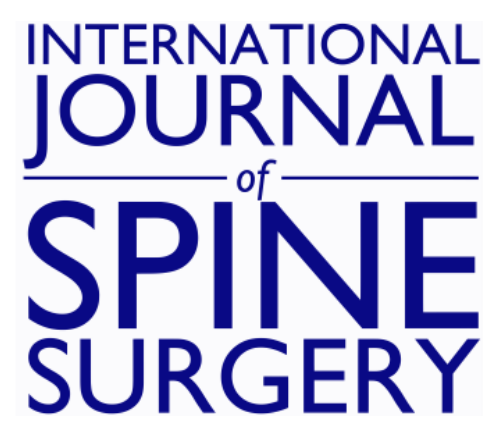

\title{
Management of Metastatic Spinal Cord Compression in Ireland: Are Surgeons Overlooked?
}

PAULA M. MCQUAIL, BEN S. MCCARTNEY, JOSEPH F. BAKER, MUTAZ JAADAN and JOHN P. MCCABE

Int J Spine Surg 2018, 12 (4) 428-433

doi: https://doi.org/10.14444/5051

http://ijssurgery.com/content/12/4/428

This information is current as of April 26, 2023.

Email Alerts Receive free email-alerts when new articles cite this article. Sign up at:

http://ijssurgery.com/alerts

The International Journal of Spine Surgery

2397 Waterbury Circle, Suite 1,

Aurora, IL 60504, Phone: +1-630-375-1432 


\title{
Management of Metastatic Spinal Cord Compression in Ireland: Are Surgeons Overlooked?
}

\author{
PAULA M. MCQUAIL, MB BCH BAO MCh MRCSI, ${ }^{1}$ BEN S. MCCARTNEY, MB BCH BAO MRSCI, ${ }^{2}$ JOSEPH \\ F. BAKER, MB BCH BAO FRSCI, ${ }^{3}$ MUTAZ JAADAN, MB BCH BAO MRSC, ${ }^{4}$ JOHN P. MCCABE, MB BCH \\ BAO FRSCI ${ }^{5}$ \\ ${ }^{I}$ Department of Trauma and Orthopaedic Surgery, Galway University Hospital Network, Ireland, ${ }^{2}$ Royal College of Surgeons Ireland, Stephens Green, Dublin, \\ Ireland, ${ }^{3}$ Department of Trauma and Orthopaedic Surgery, Galway University Hospital Network, Ireland, ${ }^{4}$ Department of Trauma and Orthopaedic Surgery, \\ Galway University Hospital Network, Ireland, ${ }^{5}$ Department of Trauma and Orthopaedic Surgery, Galway University Hospital Network, Ireland
}

\begin{abstract}
Background: The National Institute of Health and Clinical Excellence (NICE) provide a framework of evidencebased guidelines for the management of metastatic spinal cord compression (MSCC). We aimed to compare our center's provision of service to these best practice guidelines and discuss key shortcomings with their implications for the spinal surgeon.

Methods: Patients with radiologic evidence of MSCC over a 30-month period were identified using the hospital electronic radiological database. A chart review was performed analyzing MSCC management.

Results: Forty-one patients were identified. Pain was the most common presenting complaint, occurring in $76 \%$ of patients. Radiotherapy alone was the most common therapy employed (93\% of patients). A surgical opinion was sought for $51 \%$ of patients. Histological diagnosis of the causative lesion occurred in 5 patients from surgical specimens.

Conclusions: Incongruities between NICE guidelines and our practice exist. Early involvement of the spinal surgical services needs to be encouraged. Establishing a histological diagnosis of the spinal lesion should be seen as of therapeutic importance.
\end{abstract}

Other \& Special Categories

Keywords: axial metastases, cancer, metastatic spinal cord compression, radiotherapy, surgery

\section{INTRODUCTION}

Metastatic spinal cord compression (MSCC) is defined in the National Institute of Health and Clinical Excellence (NICE) guidelines as "spinal cord or cauda-equina compression by direct pressure and/or induction of vertebral collapse by metastatic spread or direct extension of malignancy that threatens neurological disability." $\mathrm{MSCC}$ is an oncological and often surgical emergency demanding immediate recognition, appropriate investigation, and timely treatment to circumvent potentially devastating neurological and functional outcomes. The true incidence and burden of MSCC in Ireland is unknown, as it is not systematically recorded by the National Cancer Registry. The incidence documented in non-Irish studies is $5-14 \%$ of cancer patients. $^{2}$ MSCC's incidence will continue to increase as treatments for primary malignancies successfully advance.

Diagnosis of MSCC can be difficult due to patient, clinician, and institutional factors. ${ }^{3}$ MSCC can present with a range of symptoms from minor sensory, motor, or autonomic disturbances to severe pain and complete paraplegia, which have enormous implications on both quality of life and prognosis. ${ }^{4}$ Nonspecific back pain is a common symptom in both the general and the oncological population. ${ }^{5}$ Furthermore, despite most cases of MSCC developing in known cancer patients, 20\% of all cases are an initial manifestation of cancer. ${ }^{6}$

Radiological investigation of MSCC is key to assisting diagnosis and clinical decision making. Excluding cord compromise in the acute setting is wholly dependent on accessibility to appropriate imaging. Magnetic resonance imaging (MRI) remains the investigation of choice for MSCC.

NICE guidelines advocate a multidisciplinary approach incorporating medical oncology, radiation oncology, and spinal surgeons in addition to the rehabilitative paramedical services in the treatment of these patients. ${ }^{1}$ Our objectives were to determine the burden of MSCC in our institution, the degree of spinal surgeon involvement in the 
Table 1. Primary malignancies affecting our patients with metastatic spinal cord compression.

\begin{tabular}{lcc}
\hline Primary Malignancy & $\begin{array}{c}\text { No. of Patients } \\
\text { Affected }\end{array}$ & Proportion (\%) \\
\hline Prostate & 11 & 26.8 \\
Lung & 11 & 26.8 \\
Breast & 6 & 14.6 \\
Colorectal & 3 & 7.3 \\
Myeloma & 2 & 4.9 \\
Lymphoma & 2 & 4.9 \\
Renal & 2 & 4.9 \\
Thyroid & 1 & 2.4 \\
Bladder & 1 & 2.4 \\
Ureteric transitional cell carcinoma & 1 & 2.4 \\
Unknown primary & 1 & 2.3 \\
\hline
\end{tabular}

assessment and treatment of patients with MSCC, and the degree to which a histological diagnosis was sought prior to commencing therapy. We then wished to assess the level of compliance that our practice in a major Irish regional cancer institution has with the recommendations on these parameters as advocated by the NICE MSCC management best practice guidelines. ${ }^{1}$

\section{METHODS}

Patients who had MRIs of their spine with the indication of investigating for spinal metastatic disease from November 2006 to April 2009 inclusive were identified from the radiological electronic database in the hospital. Inclusion criteria included patients whose radiological reports documented evidence of MSCC. Patients with documented "impending MSCC" were also included.

A retrospective chart review was carried out on the identified patients. Details collected were the clinical presentation, referral pathways, timing of involvement of a surgical service in the treatment of patients with MSCC, degree of histological diagnosis prior to commencing definitive therapy, and type of definitive treatment of these patients; specifically, radiotherapy and/or surgical intervention were carried out. We also analyzed the burden of MSCC in our institution as well as survival times in those who were deceased at the time of the study. Microsoft Excel was used to collate the data. We then examined the degree of alignment of the service we provide with NICE guidelines on management of patients with MSCC, a guiding body in the United Kingdom. NICE guidelines were used as the comparative standard, as the Republic of Ireland (ROI) does not have a national clinical guideline pertaining to MSCC care.
Table 2. Treatment modality for the 41 patients with metastatic spinal cord compression (MSCC) or threatening MSCC.

\begin{tabular}{lc}
\hline Treatment Regime & No. of Cases \\
\hline Medical therapy only & 36 \\
Surgery alone & 0 \\
Neoadjuvant therapy and surgery & 3 \\
Surgery and adjuvant therapy & 2 \\
\hline
\end{tabular}

\section{RESULTS}

Three hundred and sixty-one patients were identified as having had an MRI to investigate for MSCC in this retrospective cohort study. One hundred and seventy-one patients were identified as having metastatic spinal column disease. Of these, 41 had evidence of metastatic spinal cord compression: 26 were male, and 15 were female. The average age of the female was 64 years (standard deviation [SD] \pm 11.17 years, range 39-81) and for males was 67 years $(\mathrm{SD} \pm 8.0$ years, range $53-82$ ).

Pain was the most common presenting complaint occurring in $31(75.6 \%)$ patients. Twenty-four $(58.53 \%)$ patients experienced weakness with 12 $(29.26 \%)$ and $11(26.82 \%)$ patients reporting bowel and bladder disturbances, respectively. Despite the reported level of sphincter dysfunction, only 9 of 41 $(22 \%)$ patients had a digital rectal exam documented in their chart. The distribution of primary malignancies is shown in Table 1 .

Six patients had previous biopsy of concurrent, appendicular lesions at the time of presentation with possible MSCC. Five patients had histological diagnosis made after tissue was sent for histology at the time of definitive management with surgical decompression and stabilization. Two of these patients were subsequently diagnosed with a neoplastic lesion different to their previously diagnosed primary malignancy. No patient had a histological diagnosis of the causative spinal lesion prior to commencement of definitive therapy.

Radiotherapy alone was the most common therapy employed for patients with MSCC with $93 \%$ of patients being treated by this modality. Surgical opinion was sought in $51 \%$ of cases. Of these, only 1 patient had a documented consultation within 24 hours of presentation with MSCC. Delay in surgical referral ranged from within 24 hours to 6 weeks later. Five of the 21 patients who had a surgical consult had a referral sent after radiotherapy had already been administered. Surgical intervention occurred in 5 patients. There were no documented cases of surgical complications. The 
treatment modality pathway is outlined in Table 2. Thirty-one patients were deceased at the time of this study (76\%): 22 males and 9 females. Thirty had a documented date of death. Of these 30 , the time frame between documentation of MSCC ranged from 10 to 976 days. The average survival time from onset of MSCC was 144 days (SD \pm 209.41 days). Mean male survival was 114 days $(\mathrm{SD} \pm 140.6$ days). Mean female survival was 214 days (SD \pm 305.14 days).

\section{DISCUSSION}

MSCC is an unpredictable and challenging condition requiring multidisciplinary management to optimize outcomes and quality of life. As survival times increase with the advances in oncologic therapies, MSCC is an increasingly common serious complication that presents to our services. Service provision and management protocols, however, do not appear to have evolved to parallel the increasing incidence of this medical and surgical emergency. This is reflected by the lack of a national integrated clinical care pathway guideline in the ROI for management of patients with MSCC.

The aim of our study was to determine whether our practice in a regional cancer center reflected NICE guidelines on management of patients with MSCC, a guiding body in the United Kingdom.

We found that multiple features of our therapeutic pathway were at variance with NICE guidelines. NICE guideline 1.2.1.14 advocates that all decisions on the most appropriate combinations of treatment for pain or preventing paralysis caused by MSCC should be made by relevant spinal specialists in consultation with primary tumor site clinicians and with the full involvement of the patient. ${ }^{1}$

Treatment of MSCC incorporates surgery, radiotherapy, and chemotherapy with an accompanying appropriate rehabilitation program. Decompressive laminectomy was historically the primary treatment for MSCC. Subsequently, however, with the advent of radiotherapy, several studies found that this treatment was offering no additional benefit when compared to radiotherapy treatment alone. ${ }^{7,8}$ This was mainly because traditional laminectomy approaches limited tumor resection capacity. Often the tumor could not be surgically excised in its entirety, resulting in subsequent further spinal instability and cord compression. ${ }^{7-9}$

In the 1980s, surgical techniques and instrumentation for MSCC began to advance with the introduction of circumferential complete decompression and simultaneous spinal stabilization. Several uncontrolled surgical series reported that such surgical intervention with or without postoperative radiotherapy was superior to radiation alone. ${ }^{10-13}$ However, due to patient selection bias, poor study design and imprecise end points, the conclusion has failed to be widely accepted, and radiotherapy remained the mainstay of therapy.

This was until Patchell published the first prospective multi-institutional randomized controlled trial comparing surgery with adjuvant radiotherapy versus radiotherapy alone. ${ }^{14}$ This landmark trial yielded overwhelming results in favor of surgical intervention such that the study was stopped at interim analysis. Patients treated surgically retained and regained ambulatory function significantly more than the radiotherapy arm. Additionally, the surgical group had increased survival time and a lesser incidence of prolonged hospital stays or complications than the radiotherapy group.

Spinal surgeon's contribution to MSCC care is based on both their diagnostic and their therapeutic abilities. Spinal surgeons are essential components of the integrated care process, as they possess the most expertise in determining spinal instability, which is an indication for surgery. Instability recognition for the oncologist and other nonsurgical members of the multidisciplinary care team is more demanding, possibly resulting in underrecognition and underreferral of patients who may benefit from surgical intervention. ${ }^{15,16}$ Farther afield, the failure to consult surgeons has also led to a predilection toward initial treatment with radiotherapy despite evidence to the contrary. ${ }^{14,17}$ Our results reflect similar behavior. Surgical opinion should be sought on all patients with MSCC as per evidence-based guidelines. ${ }^{1}$

Only $51 \%(21 / 41)$ of our patients had a spinal surgical referral. Of these, only 1 patient had a documented consultation within 24 hours. These results show poor compliance with NICE guidelines. This mirrors the findings of Brooks et al, ${ }^{18}$ who, after surveying 96 trainee doctors about MSCC, concluded that there was a lack of awareness of the guidelines.

Additionally, 5 of the 21 patients had commenced a definitive nonsurgical therapy prior to spinal surgical referral. Unfortunately, this reflects previous evidence suggesting that surgical referral is 
inconsistent and regularly sought after initial radiotherapy. ${ }^{19}$ Administration of radiotherapy before surgical opinion makes potential surgery more difficult. Radiotherapy compromises the timing of potential surgery, increases technical difficulty of the procedure, and increases risk and complication rates, such as wound dehiscence and surgical site and deep tissue infection, with consequent potentially poorer outcomes. ${ }^{20}$ Therefore, early surgical referral is essential for these patients prior to commencing MSCC treatment in order to advise on the most appropriate therapeutic pathway and potentially offer surgical intervention as appropriate.

Other studies have concurred with such a recommendation, having documented the discrepancy that exists in physical examination. ${ }^{21}$ Muscle charting or utilizing the American Spinal Injury Assessment score are possible options. ${ }^{22}$

NICE guideline 1.5.3.2 advocates an "attempt to establish the primary histology of spinal metastases (including by tumor biopsy if necessary) when planning definitive treatment." Five of 41 patients had a histological diagnosis of the lesion-causing MSCC. However, the diagnostic specimen was procured at the time of definitive treatment rather than prior to this, when a histological diagnosis would be used to plan definitive therapy. Two of these patients were consequently diagnosed with a neoplastic spinal lesion of different origin to that of their previously diagnosed malignancy. This is at variance with NICE guidelines. Therapy should be based on a tissue diagnosis where possible, as this will facilitate deciding on the appropriate treatment and the predicted response to treatment and identify a vascular lesion amenable to preoperative embolization. ${ }^{15,23}$ Many differential diagnoses exist for spinal lesions, such as a vertebral abscess, Pott disease, or indeed a malignancy other than that of the already diagnosed disease. Consequently, administering radiation therapy or instigating major surgery for what is potentially a vertebral abscess or a radio-resistant tumor could have devastating consequences.

NICE guideline 1.4.3.3 recommends "MRI of the whole spine in patients with suspected MSCC unless there is a specific contra-indication." This should be done "within 24 hours in the case of spinal pain suggestive of spinal metastases and neurological symptoms or signs suggestive of MSCC, and occasionally sooner if there is a pressing need for emergency surgery."1 It was not clear from our review of the charts what proportion of our patients had an MRI performed within the recommended 24-hour window; as such, we could not comment on compliance in this regard. However, a recently published audit in another regional cancer center in the ROI demonstrated that only $50 \%$ of their MSCC patients in a 1-year period had an MRI within 24 hours of clinical suspicion. ${ }^{24}$

Despite the obvious clinical need for 24-hour availability of MRI services, Kelly et al illustrated the overwhelming lack of coordination and provision of such services in the ROI. They audited 34 public sector Irish hospitals and found that only 50\% had MRI services available from 9:00 AM to 5:00 PM with only 2 of 34 having MRI availability out of hours on a regular basis. ${ }^{25}$ Despite MRI first becoming available in 1989 in the private sector in the ROI, there remains a distinct lack of availability of this service in designated cancer centers. This is clearly at variance with NICE guidelines, and such stark deficits are of great concern when faced with appropriately managing a patient with suspected MSCC.

Ultimately, patient management is determined by service provision. Of the 8 cancer centers in Ireland, only 4 have on-site radiation oncology facilities. Furthermore, only 5 of the 8 centers employ spinal surgeons. All centers have medical oncology services within the institution. If one additionally incorporates the availability of 24-hour MRI when reviewing the current services provided, no single site provides all the necessary services to appropriately manage patients with MSCC. The deficit in trained spinal surgeons and their services, paralleled with the obvious lack of 24-hour MRI and radiation oncology facilities, highlights the grave deficits that exist in our provision of a national comprehensive system of care for patients with MSCC. ${ }^{25}$

We acknowledge that this study has limitations. As with any retrospective analysis based on medical records review, there is the potential for a deficiency of data depending on both the accuracy and the legibility of the notes. The documentation of date and time in the medical records can also be lacking, leading to a misinterpretation of exact timing. Although the health care systems in the United Kingdom and the ROI are similar in structure and the guidelines provided by NICE are likely suitable for both, the adherence to these is not necessarily a sought-after standard of care in the ROI. Assessing 
the neurologic presentation and analyzing functional outcome according to treatment paradigm could also provide valuable additional information. However, we feel that it is clear that a delay in appropriate intervention leads to poorer functional outcome, and the need here was to focus on the pathway of care rather than the end result.

\section{CONCLUSIONS}

Metastatic spinal cancer undoubtedly represents a large burden of disease in the Irish health service. However, Ireland's secondary cancer center service remains dramatically underdeveloped. Current practice in this regional cancer center does not wholly comply with NICE guidelines. We recommend the establishment of a multidisciplinary group for MSCC management in every primary cancer network with greater emphasis on surgical contribution to care. Surgical techniques have advanced such that several studies have provided evidence on the superior benefit of surgical treatment in terms of pain, survival, and ambulatory status over radiotherapy in the appropriate patient group. However, the consistency of surgical referral for patients with MSCC has failed to parallel such advancement. An early surgical referral needs to be advocated as an integral step in the management of patients with MSCC. Establishing a histological diagnosis must also be considered of therapeutic importance. Service provision in Ireland needs to be restructured to reflect the geography of the appointed cancer centers with all aspects of care being available on a single site.

\section{REFERENCES}

1. White B. Metastatic Spinal Cord Compression: Diagnosis and Management of Patients at Risk of or with Metastatic Spinal Cord Compression. NICE Clinical Guidelines, No. 75. Cardiff, Wales: National Collaborating Centre for Cancer (UK);2008.

2. Byrne TN, Benzel EC, Waxman SG. Epidural Tumors: Diseases of the Spine and Spinal Cord. Oxford, UK: Oxford University Press; 2000.

3. Abrahm JL. Assessment and treatment of patients with malignant spinal cord compression. J Support Oncol. 2004;2(5):377-388, 391; discussion 391-373, 398, 401.

4. Gilbert RW, Kim JH, Posner JB. Epidural spinal cord compression from metastatic tumor: diagnosis and treatment. Ann Neurol. 1978;3(1):40-51. https://doi.org/10.1002/ana. 410030107.

5. Loblaw DA, Perry J, Chambers A, et al. Systematic review of the diagnosis and management of malignant extradural spinal cord compression: the Cancer Care Ontario Practice Guidelines Initiative's Neuro-Oncology Disease Site
Group. J Clin Oncol. 2005;23(9):2028-2037. https://doi.org/10. 1200/JCO.2005.00.067.

6. Schiff D, O'Neill BP, Suman VJ. Spinal epidural metastasis as the initial manifestation of malignancy: clinical features and diagnostic approach. Neurology. 1997;49(2):452456.

7. Kim RY, Spencer SA, Meredith RF, et al. Extradural spinal cord compression: analysis of factors determining functional prognosis-prospective study. Radiology. 1990;176 (1):279-282. https://doi.org/10.1148/radiology.176.1.2353102.

8. Sundaresan N, Rothman A, Manhart K, et al. Surgery for solitary metastases of the spine: rationale and results of treatment. Spine (Phila Pa 1976). 2002; 27(16):1802-1806.

9. Loblaw DA, Laperriere NJ, Mackillop WJ. A populationbased study of malignant spinal cord compression in Ontario. Clin Oncol. 2003;15(4):211-217.

10. Greenberg HS, Kim JH, Posner JB. Epidural spinal cord compression from metastatic tumor: results with a new treatment protocol. Ann Neurol. 1980;8(4):361-366. https:// doi.org/10.1002/ana.410080404.

11. Siegal T, Siegal T, Robin G, et al. Anterior decompression of the spine for metastatic epidural cord compression: a promising avenue of therapy? Ann Neurol. 1982;11(1):28-34. https://doi.org/10.1002/ana.410110106.

12. Hammerberg KW. Surgical treatment of metastatic spine disease. Spine (Phila Pa 1976). 1992;17(10):1148-1153.

13. Leviov M, Dale J, Stein M, et al. The management of metastatic spinal cord compression: a radiotherapeutic success ceiling. Int J Radiat Oncol Biol Phys. 1993;27(2):231-234.

14. Patchell RA, Tibbs PA, Regine WF, et al. Direct decompressive surgical resection in the treatment of spinal cord compression caused by metastatic cancer: a randomised trial. Lancet. 2005;366(9486):643-648. https://doi.org/10.1016/S01406736(05)66954-1.

15. Eastley E, Newey M, Ashford RU. Skeletal metastasesthe role of the orthopaedic and spinal surgeon. Surg Oncol. 2012;21(3):216-222. https://doi.org/10.1016/j.suronc.2012.04. 001.

16. Galasko CS, Norris HE, Crank S. Spinal instability secondary to metastatic cancer. $J$ Bone Joint Surg. 2000;82(4):570-594.

17. Hutton J, Leung J. Treatment of spinal cord compression: are we overusing radiotherapy alone compared to surgery and radiotherapy? Asia Pac J Clin Oncol. 2013;9(2):123-128. https://doi.org/10.1111/j.1743-7563.2012.01568.x.

18. Brooks FM, Ghatahora A, Brooks MC, et al. Management of metastatic spinal cord compression: awareness of NICE guidance. Eur J Orthop Surg Traumatol. 2014;24(suppl 1):S255-S259. https://doi.org/10.1007/s00590-014-1438-8.

19. Sundaresan N, Sachdev VP, Holland JF, et al. Surgical treatment of spinal cord compression from epidural metastasis. $J$ Clin Oncol. 1995;13(9):2330-2335.

20. Ghogawala Z, Mansfield FL, Borges LF. Spinal radiation before surgical decompression adversely affects outcomes of surgery for symptomatic metastatic spinal cord compression. Spine (Phila Pa 1976). 2001;26(7):818-824.

21. Ramsey PG, Curtis JR, Paauw DS, et al. History-taking and preventive medicine skills among primary care physicians: an assessment using standardized patients. Am J Med. 1998;104(2):152-158.

22. Maynard FM Jr, Bracken MB, Creasey G, et al. 
International standards for neurological and functional classification of spinal cord injury. Spinal Cord. 1997;35(5):266-274.

23. Wedin R, Bauer HC, Wersall P. Failures after operation for skeletal metastatic lesions of long bones. Clin Orthop Relat Res. 1999;(358):128-139.

24. Sui S, Fleming JS, Kehoe M. An audit of current practice and management of metastatic spinal cord compression at a regional cancer centre. Ir Med J. 2011;104(4):111-114.

25. Kelly JC, O'Brian JE, Kelly GA, et al. Imaging the spine for tumour and trauma - a national audit of practice in Irish hospitals. The Surgeon. 2012;10(2):80-83. https://doi.org/10. 1016/j.surge.2011.01.007.

Disclosures and COI: There are no potential conflicts of interest to be disclosed by any of the authors, and a conflict of interest disclosure form has been completed by the corresponding author and is available on request. Local ethical board approval was not sought, as the Audit Department approved the process in accordance with establishing compliance with best practice. As such, informed consent was not necessary given the retrospective nature of the study. We the authors declare that we have full control of all primary data, and we agree to allow the journal to review the data if requested. No funding was obtained for this article.

Corresponding Author: Paula M. McQuail, Department of Trauma and Orthopaedic Surgery, Galway University Hospital Network, Ireland. Phone: 353 879312115; Fax: 35346 9053105; Email: paulamcquail@gmail.com.

Published 31 August 2018

This manuscript is generously published free of charge by ISASS, the International Society for the Advancement of Spine Surgery. Copyright (c) 2018 ISASS. To see more or order reprints or permissions, see http://ijssurgery.com. 